\title{
WELCOME ETHOLOGISTS TO THE SOUTHERN END OF THE WORLD
}

\author{
Ana Maria Fernandez ${ }^{1}$ José Antonio Muñoz-Reyes², Carlos Rodriguez-Sickert ${ }^{3}$ \\ 1 Universidad de Santiago de Chile \\ 2 Universidad de Playa Ancha, Valparaiso, Chile \\ 3 Universidad del Desarrollo, Santiago, Chile
}

The 24th International Society for Human Ethology (ISHE) conference in Chile 2018 was an exciting and unique opportunity to receive our fellow human ethologist at the Southern end of the world, with an amazing group of young researchers and helpers, which resulted in a real collective meeting.
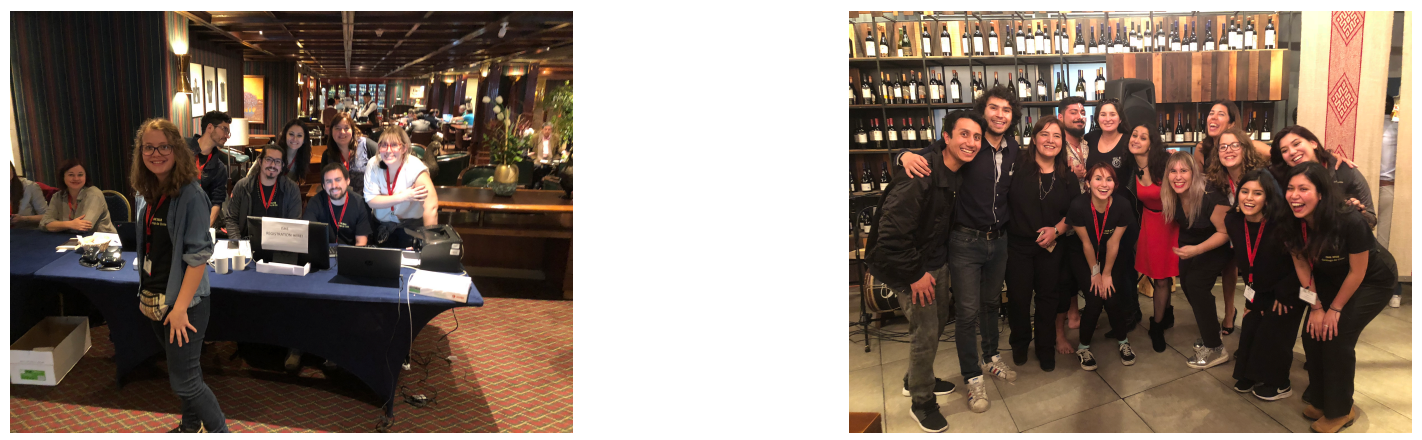
From the previous conference located at the very other extreme of the world in the North of Scotland, coming all the way to Chile had some exciting travel planning challenges, which we tried to drive away with a four day pre-conference trip to the breath taking Atacama Desert. And indeed, we had the convergence of over 17 countries in our meeting, ranging from Japan, to Australia, which made our extremely supporting and organized vicepresident from Austria, a real local!
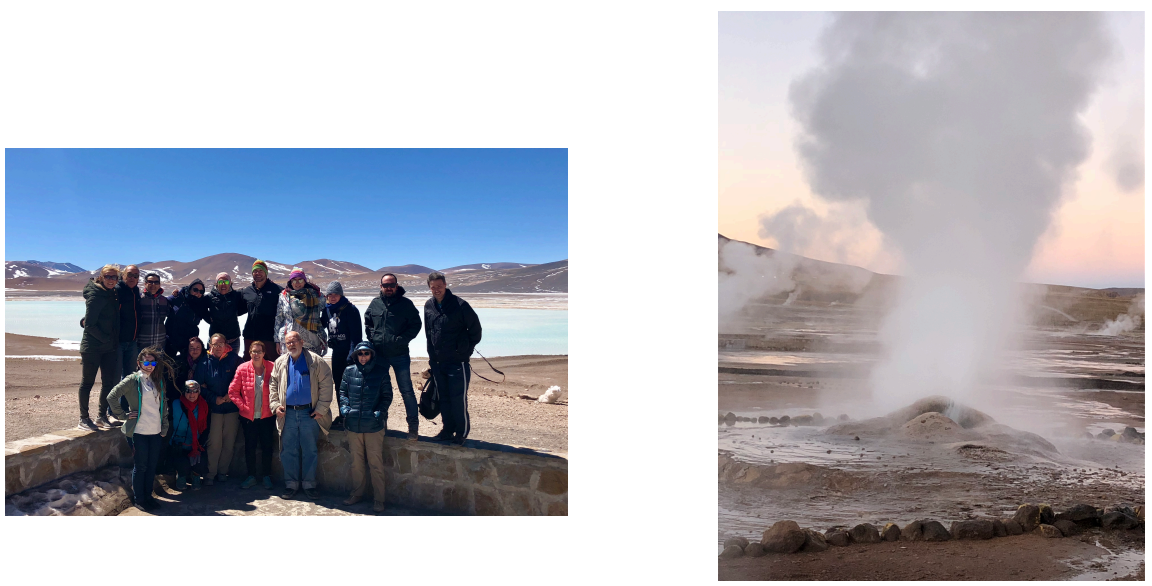

During the conference we had social gatherings hosted by the Chilean evolutionary community led by Alvaro Fisher and Ximena Katz, and our co-organizing Institutions: Universidad del Desarrollo and Universidad de Playa Ancha. We had an afternoon visit organized by our hosts at the School of Psychology to University of Santiago, which dates back over 150 years, and holds a widespread memory of the political turmoil and the dictatorship in Chile on the 70's and 80's, and followed by a visit to our Human Rights Memorial Museum. Then at night, we were delighted by the sunset at San Cristobal Hill, followed by late night dancing, which was also a highlight of our closing banquet with Rapa Nui dancing lessons.

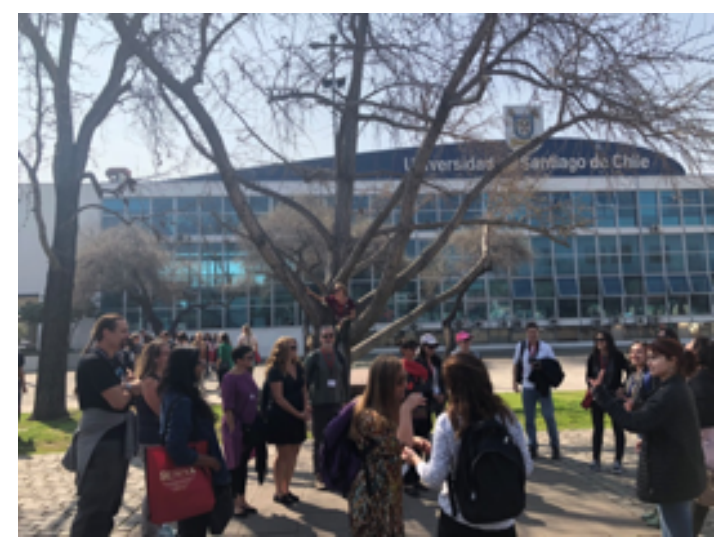



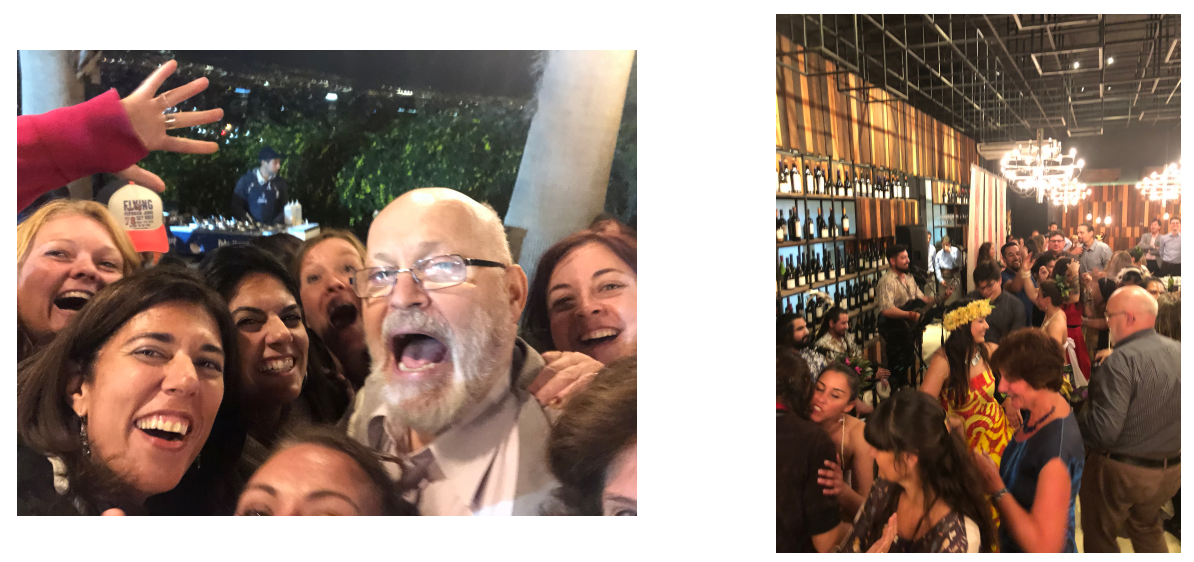

Besides the adventurous experience, the science at ISHE 2018 was also a highpoint, and great reflection of the diversity in our present society stamped on the plenaries. We began with a wonderful ethological observation of tool innovations in Galapagos birds, and continued with a detailed account of the Life History challenges and adaptations observed in the Shuar of Equatorian Amazonia. Following, we "viewed" a Game of Thrones characterized account of Incest Avoidance, and continued with gender differences analyses across cultures. Finally, a novel explanation of how mother milk is adjusted to sustain Life History challenges left us thinking how Human Ethology has spread across so many different disciplines, throughout the globe, to include such a multiplicity of arenas interested in the study of Human Behavior. Tinbergen's four levels of analysis were carefully covered by the plenaries and talks at ISHE, and in addition a memorial of Renki's (Iraneus Eibl-Eibesfeld) contribution to the field marked our Chilean conference.

Mentioning the highlights of the specific thematic sessions, awards, and posters wouldn't make justice to the variety of current Human Ethology. But these Proceedings of the ISHE 2018 Conference in Santiago, aim at documenting a sample of the diversity of research in Human Ethology that we can find in our amazing group that came to the $24^{\text {th }}$ Biennial Meeting in Chile.

So when we thought that the context of Human Ethology was all covered in planet Earth, we begin with our first article that evaluates the epigenetic changes that occur in other contexts (beyond earth!), comparing the $\mathrm{MZ}$ twin that went to space with the one that stayed in earth. During a year in which we celebrate 50 years since Humans visited the moon, Tafforin \& Segal's (2019) article point to some beautiful comparative design of the effects of real spatial experience for humans. The most provocative finding is that genetic expression is sensitive to the environmental conditions astronauts face in space. These results are particularly relevant now that humanity has Mars in its sights. A round-trip journey will minimally require a 6-month outward trip and a 6-month return trip, while a stay on the red 
planet could last for one year and beyond. Epigenetic changes will impact human behavior at both the individual and at the social level: genetic expression resulting from a weightlessness environment, through adaptive behavioral strategies, will yield a new cognitive representation of the members of a space team. These changes should be taken into account in any design for such monumental enterprise.

Neither theory nor methodology were absent from the Proceedings. Santana (2019) develops a theoretical review and a conceptual analysis of William James work, which has almost been exclusively the subject of Psychology, but now his insights are brought to the field of Human Ethology recognizing the mind and thought distinctions as sources of variability in the behavior of organisms that has been overlooked. Prosssinger, Schmehl and Oberzaucher (2019), on the other hand, propose a methodology, based on Bayesian statistics which can be used to build gesture dictionaries and thereby enhance the statistical reliability of ethologists' encoding systems, including, for example, those for facial expressions.

Lucci, DeFelipe, Andrade, David and Otta (2019), go back to the very basis of Human Ethology by coding the first minutes of new born contact with their mothers, uncovering sex differences as well as other particularities of this sample of Brazilian newborn's crying and mother-child interaction that may later affect attachment behavior.

And finally, an account of touching behavior in Eder \& Oberzaucher's (2019) work, presents an interesting and innovative approach to analyze the effect of status in interactions in 10-13 years old students. Results show that touching of intimate zones frequently involves individuals with extremely high or low status, particularly boys in mixed-sex dyads. On the contrary, same-sex touching of intimate zones occurs more frequently among individuals of similar status, which leads to considering that touching serves a different function for high or low status individuals, and affects their position in their social networks.

To conclude, we must mention that these Proceedings would have not been possible without the patience and support of the Human Ethology Bulletin team, thank you Colin, Kathi and Susanne. ISHE 2018 was a collective conference and these proceedings are also a group effort lead by the Editor of the Human Ethology Bulletin. Thank you ISHE for coming to Chile!

\section{REFERENCES}

Eder, S. J. \& Oberzaucher, E. (2019). Getting in Touch - Social status predicts physical interaction in classrooms. Human Ethology, 34(1), 159-172. DOI 
Lucci, T.K., Defelipe, R.P., Andrade, N.C., David, V.F., Otta, E. (2019). Early mother-infant interaction after delivery: A naturalistic study. Human Ethology, 34(1), 130-143. DOI

Prossinger, H., Schmehl, S., Oberzaucher, E. (2019). Statistical Analysis of Gesture Encoding: How consistently can ethologists encode what they observe? Human Ethology, 34(1), 173-193. $\underline{\mathrm{DOI}}$

Santana, L.H. (2019). Evolutionary Ideas and the Naturalization of Mind in William James. Human Ethology, 34(1), 144-158. DOI

Tafforin, C. \& Segal, N.L. (2019). Twins in Space: Review and Perspectives. Human Ethology, 34(1), 123-129. DOI 